\title{
A Novel Path-Loss Model for UWB Off-Body Propagation
}

\author{
Angelos A. Goulianos, Tim W. C. Brown, and Stavros Stavrou, Member, IEEE
}

\begin{abstract}
A novel, multi-slope dual breakpoint model for predicting path-loss in Ultra-Wideband (UWB) off-body communication channels, is proposed. This model is based on real-body measurements, carried out in the frequency range between $3.5 \mathrm{GHz}-6.5 \mathrm{GHz}$, in an anechoic chamber. New parameters that describe this specific propagation environment are presented and evaluated. Results show that the first breakpoint point angle lies in the lit region of the transmitter and increases exponentially with distance until it rises to its threshold value. Based on this finding the near and far field areas for BAP (Body to Access Point) channels are defined. In addition, newly estimated decay coefficients suggest severe degradation as the receiver moves in between the two critical angles. Finally, techniques for model expansion in two dimensions are discussed.
\end{abstract}

Index Terms-Access Point (AP), Off-Body Communications, Path-loss model, Wireless Body Area Networks (WBAN), Ultrawideband (UWB).

\section{INTRODUCTION}

$\mathrm{U}$ Jtra-Wideband (UWB) communications, is a new transmission technology, promising a series of important advantages[1], such as insignificant multipath fading [2], possibility of extremely high data rates [3] and low power operation. This leads to UWB being a potential candidate for body-centric networks [4].

Wireless Body Area Networks (WBAN) can be classified as on-body, in-body and off-body systems [5]. To optimize receiver structures and system design of UWB-WBAN, channel modeling is essential for each of these systems.

So far though, channel characterization for communications of this sort, is mostly concentrated on studying the on-body scenario [6]-[10]. In [11] and [12] some preliminary measurements are presented for UWB off-body propagation. However, no complete path loss model is provided, and key model parameters are not investigated.

In this paper, the above issue is addressed, by developing a novel two dimensional statistical-based path-loss model, physically motivated by creeping waves propagation around the body. This model describes channel alternations with respect to body-based orientation angle, distance between AP and body, and transmitter-based azimuth angle.

Manuscript received September 19, 2007

A. A. Goulianos, T. W. C. Brown, and S.Stavrou are with the Center for Communication System Research, GU2 7XH,Surrey, Guildford, United Kingdom (e-mail: A. Goulianos, T. Brown, S. Stavrou@surrey.ac.uk).
The rest of the paper is organized as follows: Section II presents the measurement techniques, and scenarios under investigation. Furthermore, principal and body-centric coordinate systems PCS, BSC are introduced. Section III provides model development, focusing mostly on parameterization. In Section IV the new path-loss model is established and evaluated. Furthermore, the two dimensional path-loss expansion in the principal polar coordinates is discussed. Finally, Section V concludes the paper.

\section{MEASUREMENT SET-UP}

All of the results presented in this work are the outcome of measurements that took place in an anechoic chamber, where absorbers prevent secondary radiation sources, surrounding the body, from affecting the pure communication channel between the wireless terminal and the human body. Consequently, the output of this model preserves symmetries which as it will be shown later on, are very important for the description and the adequate understanding of the UWB-BAP propagation channel.

A Vector Network Analyzer (VNA) was used in measuring the channel complex frequency response in the frequency range between 3.5 and $6.5 \mathrm{GHz}$. Measurements of this sort were realized, by transmitting $\mathrm{CW}$ tones of equal power, at 801 equally spaced frequency intervals of $3.75 \mathrm{MHz}$. In the presented set-up two small-size, low profile SkyCross SMT3T010M antennas were used [6]. These antennas have omnidirectional patterns in the $\varphi$-plane and vertical z-directed electric field polarization vectors. A more detailed description of some helpful measurement tools can be found in [12].

Figure 1 illustrates the basic concept followed in the experimental work. R represents the average body equivalent radius, assuming that the periphery of the person under test is cylindrical. Four different human bodies were measured and more than 800 channel profiles were recorded. As mentioned earlier, the transmitter is mounted on a fixed point and without loss of generality this point is defined as the origin of the principal coordinate system PCS. PCS describes the position of the point $\mathrm{O}$, in the $(\mathrm{x}, \mathrm{y}, \mathrm{z})$ space for rectangular, or in the $(\rho, \varphi, z)$ space for cylindrical coordinates. Since the channel is studied for distances bigger than $\mathrm{R}$, the magnitude of the radial coordinate will always be (fig. 1 ),

$$
\rho=\rho_{C}-R
$$

The point $\mathrm{O}$ is defined as the centre of the cylindrical body- 
centric coordinate system (BCS), which is responsible for positioning the receiver with respect to the azimuth angle $\left\{\theta_{\mathrm{A}}\right\}$. This angle is formed by the line connecting $\mathrm{O}$ to the onbody sensor and the parallel to $\mathrm{x}$-axis line segment, which crosses $\mathrm{O}$. At this point, please note that in figure 1, cylinders represent the human bodies used in our real chamber measurements. Each measured body is rotated around the symmetrical axis $z$, from $0-360$ degrees in equally spaced steps of 18 degrees, with the aid of a mechanical turntable which is controlled by an efficient computer program.

It is apparent by carefully looking at fig.1, that 21 BCSbased $\mathrm{R}_{\mathrm{xi}}$ measured positions correspond to each one of the PCS-based $A_{k}$ positions. The ten $A_{k}$ positions are equally spaced along the $\mathrm{x}$-axis and cover a distance between 0.3 and 3 meters.

It also has to be mentioned, that the transmitter and the receiver heights are equal, which means that the condition $\{\Delta \mathrm{z}=0\}$, where $\Delta \mathrm{z}$ is the height difference between transmitter and receiver, is applied in all measurements

\section{ANALYSIS}

\section{A. The Multiple-Slope BAP Channel Model}

Careful consideration of the BAP channel characteristics prompts us to model the off-body communication channel with a multiple-slope linear path-loss equation in the semilogarithmic domain. A linear semi-logarithmic decay model is equivalent to an exponential decay model which is in accordance with the fact that creeping waves are attenuated exponentially with the body orientation angle $\theta_{\mathrm{A}}$, since they travel around a convex conducting cylindrical surface [13].

Multiple slopes are expected to appear since link performance between the access point and the on-body sensor node is strongly affected by any change of the angle $\theta_{\mathrm{A}}$. As long as $\theta_{\mathrm{A}}$ is such that a Line of Sight (LoS) link is maintained and the antenna radiation pattern is not distorted by the body rotation, path loss exponent value is expected to be low. On the other hand, as the rotation angle increases the on-body node lies in the shadow zone where it is expected that the link will suffer deep degradation. In addition, as the observation point enters deeper into the shadow region, the constructive and destructive interference caused by the clock-wise and anticlockwise waves around the body results in stabilization of the channel loss in that area.Mathematically this model can be expressed as:

$$
L\left(\rho, \theta_{A}\right)=\left\{\begin{array}{cc}
L_{0}(\rho)-n_{\theta}\left(\theta_{A}\right)\left(\theta_{A}-\theta_{0}\right), & 0 \leq \theta_{A} \leq \theta_{A L}(\rho) \\
L\left(\theta_{A L}\right)-n_{\theta}\left(\theta_{A}\right)\left[\theta_{A}-\theta_{A L}(\rho)\right], & \theta_{A L}(\rho) \leq \theta_{A} \leq \theta_{A S}(\rho) \\
L\left(\theta_{A S}\right)-n_{\theta}\left(\theta_{A}\right)\left[\theta_{A}-\theta_{A S}(\rho)\right], & \theta_{A S}(\rho) \leq \theta_{A} \leq \pi
\end{array}\right.
$$

In the equation above, $\left\{\theta_{\mathrm{AL}}\right\}$ denotes the first breakpoint angle, observed in the lit region of the transmitter. Similarly, $\theta_{\mathrm{AS}}$, denotes the shadow-region breakpoint angle which is observed in the shadow region of the transmitter. Azimuth decay coefficients are expressed by $\mathrm{n}_{\theta}\left(\theta_{\mathrm{A}}\right)$ and in general they have different values within each of the angle intervals given by (2). Furthermore, $\mathrm{L}_{0}(\rho)$ represents the channel loss when $\left(\theta_{\mathrm{A}}=\theta_{0}=0\right)$. Therefore, since path loss towards the radial direction is a function of distance only, it is expressed similarly to [14] as:

$$
L_{0}(\rho)=L_{0}\left(\rho_{0}\right)+10 n_{\rho} \log _{10}\left(\frac{\rho}{\rho_{0}}\right)
$$

where $\mathrm{L}_{0}\left(\rho_{0}\right)$ is the reference path loss, measured at a distance $\rho_{0}=0.3 \mathrm{~m}$ from the body surface, $\mathrm{n}_{\rho}$ is the radial decay coefficient and $\rho$ is the distance between the two antennas.

\section{B. Breakpoint positioning and human body effects}

A breakpoint angle, is defined as the angle after which the decay coefficient $\left\{n_{\theta}\left(\theta_{A}\right)=\frac{\partial L(\rho, \theta)_{d B}}{\partial \theta}\right\}$, change its arithmetic value. In the proposed model two breakpoint angles are observed with each one representing the pass from one region to another as described in (2). These regions are defined similar to [7] as front, side and back of the body. Moreover, initially we assume that both breakpoint angles are functions of distance $\rho$, although we will prove in Section IV that only the first critical angle depends on it.

Relating $\theta_{\mathrm{AL}}$ to distance requires knowledge of the boundary angle $\theta_{\mathrm{AB}}$-fig. 2- which depends on the distance between the transmitter and the BCS central point. Under ideal conditions, namely under the assumption that for any LoS scenario, antenna reception is not affected by the human body rotation and therefore maintains its free-space omnidirectional characteristics, $\theta_{\mathrm{AB}}$ is equivalent to the first breakpoint angle $\theta_{\mathrm{AL}}$ since it defines the attachment points $\mathrm{A}$ and $\mathrm{B}$. These points lie on the straight line that distinguishes observation points into the lit and the shadow region. Consequently, as the arguments of $\theta_{\mathrm{A}}$ remain smaller than $\theta_{\mathrm{AB}}$, the observation points lie within the lit region and decay coefficient $n_{\theta}\left(\theta_{A}\right)$ is expected to have low arithmetic value, whereas as the argument of $\theta_{\mathrm{A}}$ becomes bigger than $\left\{\theta_{\mathrm{AB}}\right\}$ the observation points lie within the shadow region and severe channel degradation is likely to be observed.

As the transmitter moves from the first to the n-th position, the ratio $\frac{R}{\rho}$ becomes much smaller than unity. Therefore, from the geometry of fig. 2

$$
\theta_{A B}(\rho)=\arccos \left(\frac{R}{\rho+R}\right)
$$

The maximum value of $\theta_{\mathrm{AB}}$ is found as

$$
\max \left\{\arg \left(\theta_{A B}\right)\right\}=\lim _{\rho \rightarrow \infty} \theta_{A B}(\rho)=\frac{\pi}{2}
$$

Previous analysis is useful in determining the theoretical bound of the ideal breakpoint location in any BAP channel. In 
order to investigate if ideal conditions are still valid when the receiver is mounted on the body, a new measurement set-up is used, in which the body is rotated around the vertical axis $\mathrm{Z}$ from $0^{\circ}$ to $90^{\circ}$ so that the antenna remain at the same position $\mathrm{O}_{1}$. Using this method the LoS link is maintained and the effects that different body positions have on the antenna omnidirectional pattern can be extracted.

Measurements for this scenario were carried out at a distance of 3 meters, where (5) is valid. It is apparent though (Fig. 3) that channel degradation is observed at approximately $60^{\circ}$ and not at $90^{\circ}$ as ideal conditions impose. This is an indication that the maximum breakpoint position $\theta_{\mathrm{AL}}$ in BAP channels is not given by (5) but as we will see in the next section it is exponentially related to distance $\rho$.

\section{RESULTS}

\section{A. Channel Model Parameter Estimation}

Based on the measured data and following the analysis of the previous section, statistical parameters for the proposed path loss model as expressed in (2) and (3) are obtained. Similarly to the post-processing steps referred in [15] three straight lines are fitted, each one denoting an exponential decay in linear power, to model path-loss for all BAP scenarios. Using the measured complex frequency response data $G\left(f_{i}, \rho, \theta_{A}\right)$ the empirical path loss, $\mathrm{L}_{\mathrm{e}}$, is estimated at any point $\left(\rho, \theta_{\mathrm{A}}\right)$ by performing the following on [14]

$$
L_{e}\left(\rho, \theta_{A}\right)=\frac{1}{M N} \sum_{i=1}^{N} \sum_{j=1}^{M}\left|G_{j}\left(f_{i}, \rho, \theta_{A}\right)\right|^{2}
$$

where $\mathrm{N}$ is the number of frequency tones used during the measurements, and $\mathrm{M}$ is the number of frequency-response snapshots collected at each point.

Model parameters are estimated by minimizing the mean square error between theoretical and empirical data. Figure 4 illustrates lit-region breakpoint angle variations, with respect to distance. The presented result suggests that $\theta_{\mathrm{AL}}$ increases exponentially as the radial distance between the access point and the BCS central point $\{\mathrm{O}\}$ increases. Mathematically this model is expressed as follows

$$
\theta_{A L}(\rho)=\theta_{T}\left(1-e^{-\frac{k \rho}{R}}\right)
$$

where $\theta_{\mathrm{T}}\left(\theta_{\mathrm{T}}=58^{0}\right)$ denotes the threshold value of the first breakpoint angle, and $\mathrm{R}(\mathrm{R}=15 \mathrm{~cm})$, represents the average radius of the human bodies used in our measurements. Parameter $\mathrm{k}$ has a constant arithmetic value $(\mathrm{k}=0.68)$. It is apparent from Eq. (7) that $(1 / \mathrm{k})$ denotes the value of the ratio $a_{\rho}=\left(\frac{\rho}{R}\right)$ for which $\theta_{\mathrm{AL}}$ rises to $37 \%$ of its final value. Consequently, when $\alpha_{\rho}$ becomes equal or larger than five times the inverse of $\mathrm{k}, \theta_{\mathrm{AL}}$ will have essentially risen to its final value $\theta_{\mathrm{T}}$. Mathematically the above condition is represented as

$\rho \geq \frac{5 R}{k}, \quad$ with $\rho_{f}=\frac{5 R}{k}$

Therefore, it is defined that at any distance, bigger than $\rho_{\mathrm{f}}$ the body is in the far field of the access point, whereas for smaller distances the body will be assumed to be in the AP near field.

Another important model parameter is the azimuth decay coefficient $\mathrm{n}_{\theta}$. Data analysis suggests that in each of the angle intervals in (2), $\mathrm{n}_{\theta}$ is normally distributed - $\mathrm{n}_{\theta}: \mathrm{N}(\mu, \sigma)-$. Specifically, within the front and back of the body, as defined in Section III, channel degradation is not severe and as a result, statistical processing shows that mean $\{\mu\}$ as well as standard deviation $\sigma$ of $\mathrm{n}_{\theta}$ - in- $(\mathrm{dB} / \mathrm{rad})$ - is negligible. Furthermore, at all angle intervals, standard deviation is found small compared to the mean of $\mathrm{n}_{\theta}$ and for modeling purposes can be omitted. Therefore, without loss of accuracy the following simplification can be applied

$$
n_{\theta}\left(\theta_{A}\right)=\left\{\begin{array}{cc}
N(1.1,0.33) \approx 0 & 0 \leq \theta_{A} \leq \theta_{A L}(\rho) \\
N(19.3,1.12) \approx 19.3 & \theta_{A L}(\rho) \leq \theta_{A} \leq \theta_{A S} \\
N(1.46,0.27) \approx 0 & \theta_{A S} \leq \theta_{A} \leq \pi
\end{array}\right.
$$

All other model parameters, as presented in equations (2) and (3), are independent from both the radial distance and the body orientation angle. Radial decay coefficient $n_{\rho}$ is found to have a mean value of 1.7 and its standard deviation is 0.23 . Similarly, all complementary parameters can be modeled as normally distributed random variables and their values are depicted in table I.

In fig. 5, the statistical model resulting from the postprocessing analysis by using the mean value of each parameter is compared to the corresponding empirical data. Results suggest that the proposed model provides accurate path loss predictions for UWB-BAP communication channels

\section{B. 2-D Generalization in Principal Polar Coordinates}

So far, this model describes path loss variations with respect to $\{\rho\}$ and $\left\{\theta_{\mathrm{A}}\right\}$.

TABLE I

COMPLEMENTARY CHANNEL MODEL PARAMETERS

\begin{tabular}{|c|c|c|}
\hline Parameter & $\mu$ & $\sigma$ \\
\hline$\theta_{\mathrm{AS}}$ & 139.3 & 3.7 \\
\hline $\mathrm{n}_{\rho}$ & 1.7 & 0.23 \\
\hline $\mathrm{L}_{0}\left(\rho_{0}\right)$ & -38 & 1.4 \\
\hline
\end{tabular}

However as mentioned in Section II, in all measurements the radial coordinate coincides with $\mathrm{x}$-axis direction (i.e $\varphi=0)$. Figure 6 depicts the BAP system configuration when the azimuth component of the principal coordinate system $\varphi$ is non-zero. Let $\mathrm{L}_{\varphi}$ be the channel path-loss for any non-zero $\varphi$ value and let $\mathrm{L}$ is given by Eq. (2). Then, as inferred from the 
geometry of fig. (6), path loss at each of the possible receiver positions satisfies the following equation

$$
L_{\phi}=L_{R, 2}\left(\rho, \phi, \theta_{A}\right)=L_{R d 1}\left\{\rho, 0,\left(\phi+\theta_{A}\right)\right\}=L\left\{\left(\rho,\left(\phi+\theta_{A}\right)\right\}\right.
$$

Furthermore, due to the symmetry of the human body with respect to z-axis (fig. 1), we have

$$
L\left(\rho, \theta_{A}\right)=L\left(\rho, 2 \pi-\theta_{A}\right)
$$

Therefore, determination of model parameters and implementation of (2), (3), (10) and (11) allows two dimensional path loss predictions in the principal coordinate system.

\section{CONCLUSION}

We have presented a new approach for modeling the UWB off-body communication channel. A large number of measurements were carried out in an anechoic chamber in order to evaluate the pure body to access point UWB channel. A statistical-based dual breakpoint model seems to adequately describe the path loss trend with respect to both distance and body orientation. New channel parameters have been defined and estimated by means of curve and least squares fitting analysis.

The proposed model can be used for modeling indoor sparse UWB-BAP channels. Following that the presented modeling method is general, model parameters can be used as criterion for characterization of respective dense multipath channels. Therefore, future work will first focus on this task whereas the presented model will be extended to three dimensions, taking into consideration the difference in height between access point and on-body sensor.

\section{REFERENCES}

[1] A. F. Molisch, Ultra-Wideband Propagation Channels- Theory, Measurement, and Modeling," IEEE Trans.On Vehicular Techn, vol. 54, no. 5, pp. 1528-1543, Sep. 2005

[2] M. Z. Win and R. A. Scholtz, "On the Energy Capture of Ultra-wide bandwidth signals in dense multipath environments," IEEE Comm.. Lett, vol. 2, pp. 245- 247, Sep. 1998.

[3] A. Batra et al, "Multi-band OFDM Physical Layer Proposal, Document IEEE 802.15-03/267r2, 2003.
[4] A. Alomainy, Y.Hao, X.Hu, C. G. Parini and P. S. Hall, “ UWB on-body Radio Propagation and System Modeling for Wreless Body Centric Netwoks," IEE Proc-Commun, vol. 153, no.1, pp. 107-14, Feb.2006.

[5] P. S. Hall, Y.Hao, "Antennas and Propagation for Body-Centric Wireless Communications," ${ }^{\text {st }}$ Edition, Artech House, 2006.

[6] A. Fort et al, "Ultra-Wideband channel model for communication around the body". IEEE Journal on Selected Areas in Communications, vol. 24, no.4, pp. 927-933, April 2006.

[7] A. Fort et al, "An Ultra-Widebamd Body Area Propagation Channel Model-From Statistics to Implementation,", IEEE Trans. On Microwave Theory and Techniques, vol. 54, no. 4, pp. 1820-1826, April 2006.

[8] Y. Zhao, Y. Hao, A. Alomainy and C. Parini, " UWB On-Body Radio Channel Modeling Using Ray Theory and Sub-band FDTD Method," IEEE Trans. On Microwave Theory and Techniques, vol. 54, no. 4, April 2006.

[9] T. Zasowski, G. Meyer, F. Althaus and A. Wittneben, "UWB Signal Propagation at the Human Head," IEEE Trans. On Microwave Theory and Techniques, vol. 54, no. 4, pp. 1-9, April. 2006

[10] A. A. Goulianos and S.Stavrou, "UWB Path Arrival Times in Body Area Networks," IEEE Antennas and Wireless Prop. Letters, vol. 6, pp. 223226, 2007

[11] T. B. Welch et al, "The effects of the Human Body on UWB Signal Propagation in an Indoor Environment," IEEE Journal on Selected Areas in Communication, vol. 20, no. 9, pp. 1778-1782, Dec. 2002.

[12] M. Presser, T. Brown, A. Goulianos, S.Stavrou and R. Tafazzoli, "Body Centric Aware Application Scenarios," IET Seminar on Antennas and Prop. For Body-Centric Wireless Communications, pp. 19-23, April. 2007.

[13] D. A. Mcnamara, C. Pistorius, J. Malherbe, "Introduction to the Uniform Geometrical Theory of Diffraction," Artech House: Boston, 1991.

[14] S. S. Ghassemzadeh, et al, "Measurement and Modeling of an UltraWide Bandwidth Indoor Channel," IEEE Trans. On Communications, vol. 52, no. 10, Oct. 2004.

[15] J. Ryckaert et al, "Channel Model For Wireless Communication Around Human Body," Electronic Letters, vol. 40, no. 9, pp. 543-544, April 2004.

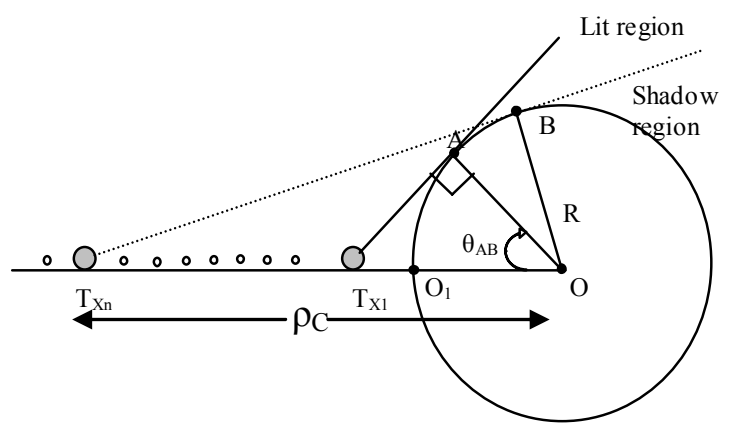

Fig. 2. Shadow boundary location with respect to distance, $\rho$. - Two dimensional top view-. 


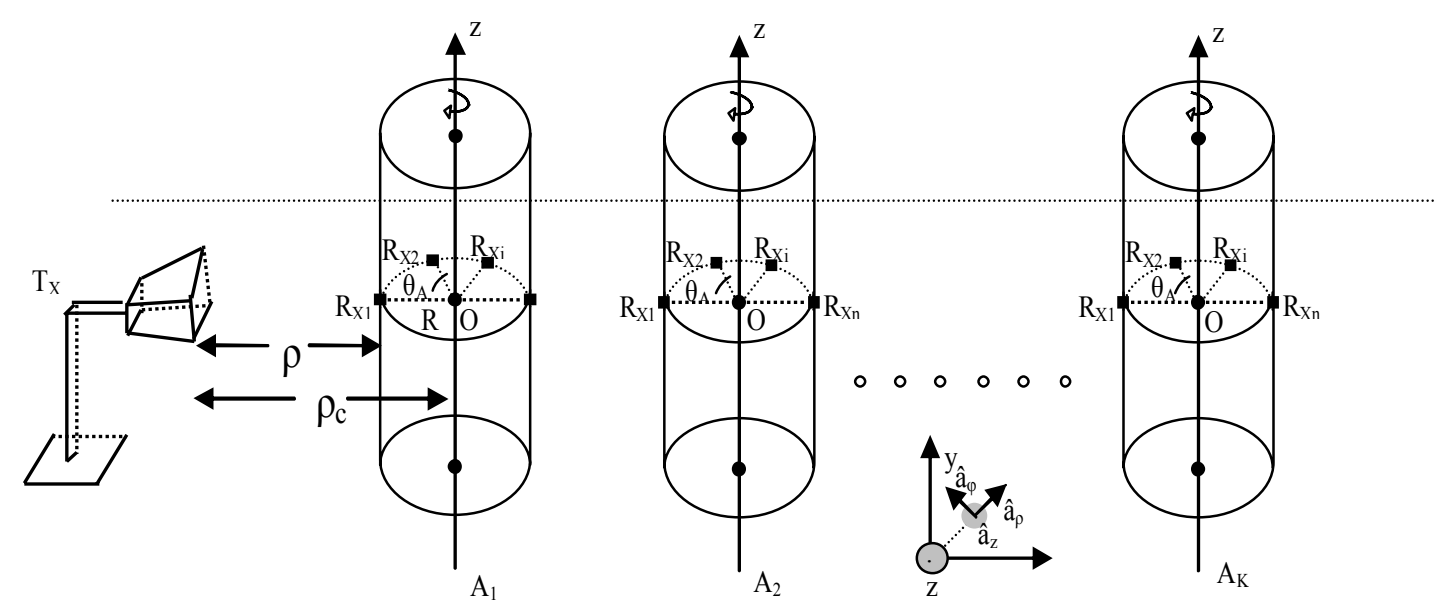

Fig. 1 Fundamental measurement set-up and, for the description of BAP communication links

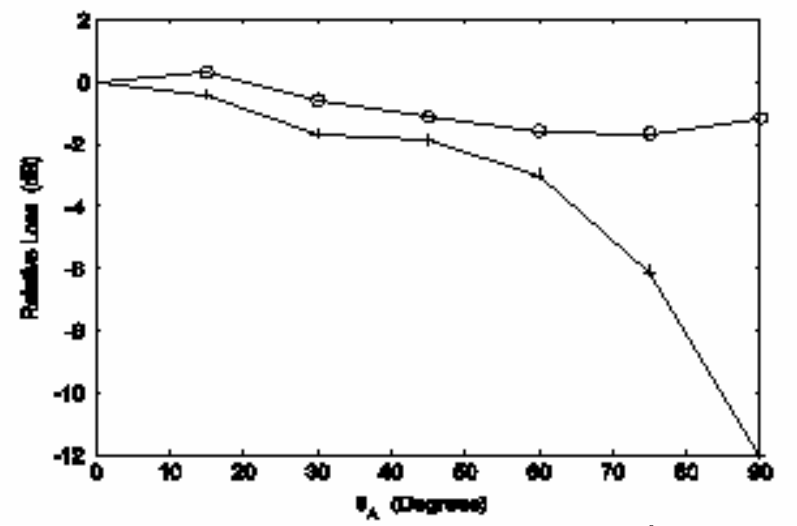

Fig. 3. Relative loss with respect to the azimuth angle $\theta_{\mathrm{A}}=0^{0}$ at $\rho=3 \mathrm{~m}$. Circles illustrate free space loss reception whereas crosses result when the body is rotated around $\mathrm{O}_{1}$.

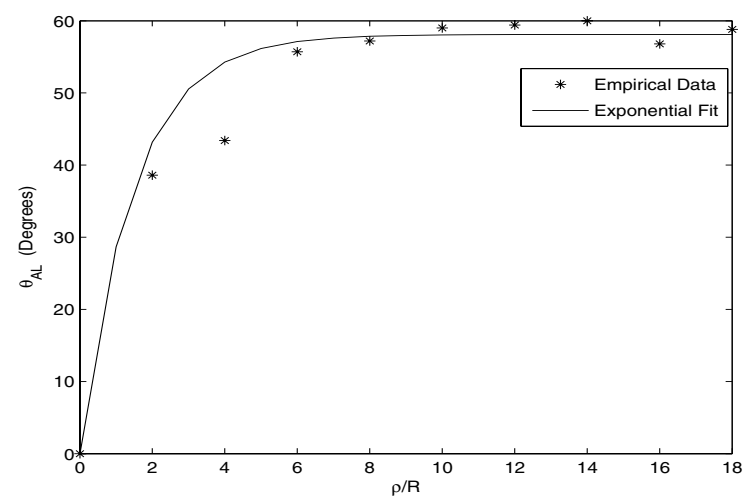

Fig. 4 Lit-region breakpoint angle $\theta_{\mathrm{AL}}$ plot as a function of the ratio of radial distance $\rho$ to the average cylindrical body radius (R).

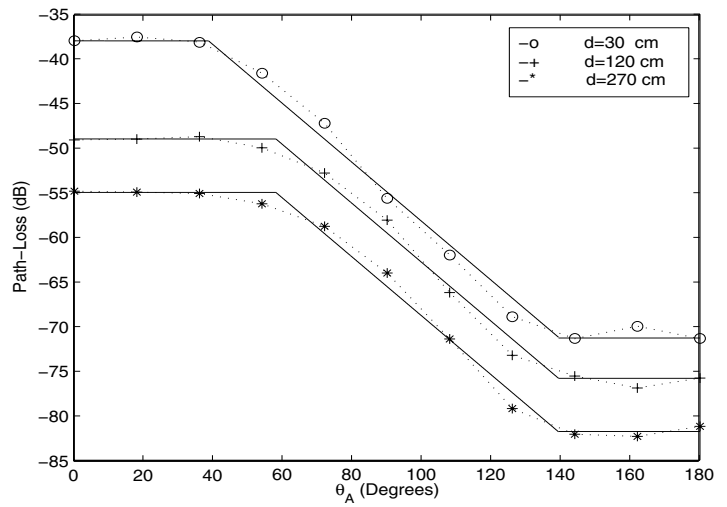

Fig. 5 Path-Loss as a function of the body orientation angle $\left(\theta_{\mathrm{A}}\right)$. Symbols represent the average empirical data whereas solid lines represent theoretical values based on the proposed channel model

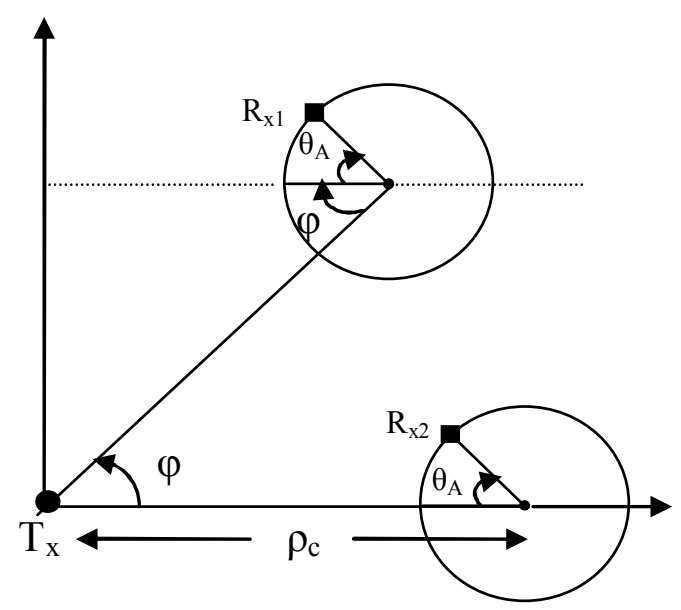

Fig. 6 Two dimensional system topology. Path-Loss at each of the receivers, depends on the azimuth coordinate $\varphi$. 